\title{
Nitrous Oxide "Whippit" Abuse Presenting with Cobalamin Responsive Psychosis
}

\author{
Nitin K. Sethi, MD, Paul Mullin, MD, Josh Torgovnick, MD, Giuseppe Capasso, Medical Student
}

Department of Neurology, Saint Vincent's Hospital and Medical Centers, New York, NY

\begin{abstract}
Introduction: Nitrous oxide is a commonly abused inhalant drug. It is known to precipitate vitamin B12 deficiency when used chronically and acutely, especially in people having marginal vitamin B12 reserve.

Case report: We are presenting a case of nitrous oxide "whippit" abuse in a thirty-three-year-old male with bizarre behavior and delusions. The patient had low normal levels of vitamin B12 (202 pg/ml), but he had markedly high methylmalonyl CoA $(1078 \mathrm{nmol} / \mathrm{L})$ and homocysteine $(48.4 \mathrm{mcmol} / \mathrm{L})$. Treatment with intramuscular cobalamin led to a resolution of his psychosis.

Discussion: This case report highlights the need to consider vitamin B12 deficiency in patients who arrive at a hospital with psychiatric manifestations and who report having a history of nitrous oxide exposure or abuse in the recent or remote past. A review of the literature on the neuropsychological manifestations of vitamin B12 deficiency is provided.
\end{abstract}

\section{INTRODUCTION}

Vitamin B12 is a member of the vitamin B complex. It is a complex molecule composed of a cobalt atom contained in a corrin ring and serves an important role in the formation of red blood cells and the maintenance of a healthy nervous system [1].

Nitrous oxide, also known as dinitrogen oxide or dinitrogen monoxide, is a chemical compound with the formula $\mathrm{N}_{2} \mathrm{O}$. It is a colorless non-flammable gas, with a slightly sweet odor, used in surgery and dentistry for its anesthetic and analgesic effects. The drug established moderate popularity as an inhalant in the United States psychedelic community. It is known to precipitate vitamin B12 deficiency when used on regular basis and acutely, particularly in people having marginal vitamin B12 reserve [2]. Nitrous Oxide causes inactivation of vitamin B12 by the oxidation of Cob (I) alamin to Cob (III) alamin and can cause functional vitamin B12 deficiency.

\section{CASE REPORT}

A woman brought her husband, a 33-year-old unemployed Indian-American male with no past psychiatric history, to the hospital because of his bizarre behavior and delusions. The patient believed that he was part of an experiment for NASA in which he serves as an "interface" between humans and machines. The patient noted commands, and he stated that he felt compelled and controlled when he destroyed furniture and broke a window at his home. He also reportedly rode his bike into a moving vehicle because his mind commanded him to do so. The patient stated that the actions were part of his "training" but that he was unable to identify the purpose of his training. He also stated that he had been told that he had a second wife and must therefore kill his current wife. The patient and his wife reported that the patient never had similar thoughts or delusions in the past. He worked as a medical technologist in the past, and he denied occupational exposure to any toxic substance.

Corresponding Author: Nitin K. Sethi, MD, Chief Resident, Department of Neurology, Saint Vincent's Hospital and Medical Centers, 170 West $12^{\text {th }}$ Street, New York, NY 10011. Email: sethinitinmd@hotmail.com 
He was in a monogamous relationship with his wife and had no children. He initially denied use of any recreational drugs and alcohol but on further questioning reported excessive use of inhalants, namely, nitrous oxide. He reportedly bought several cases of nitrous oxide containers, also known as "whippits," from a cookware store. Using a plastic container fitted with an airtight facemask, he inhaled the nitrous oxide on a daily basis for nearly 4 weeks prior to his current hospitalization. He denied any auditory or visual hallucinations, or any suicidal ideations. Sleep and appetite were reported as normal and no stressors were identified. Neurological consultation was requested to rule out any organic cause of his delusions.

An examination revealed a young man in no apparent respiratory distress. He was oriented to person, place, and time with fluent speech and intact comprehension and repetition. The patient exhibited fixed delusions as a participant in a NASA experiment. His episodic and remote memory was intact and there was no ophthamoplegia or nystagmus. All cranial nerves were intact. No motor or sensory deficits were identified, and position and vibration sensations were intact. He had no gait difficulties; a Romberg test was bilaterally normal with flexor plantar reflexes. A negative urine toxicology screen did not indicate any other commonly abused drugs such as cocaine and amphetamine. The thyroid profile was normal and he tested non-reactive for serological tests of syphilis and HIV. Taking the history of nitrous oxide abuse into consideration, we decided to send out a serum $\mathrm{B} 12$ level-even though his $\mathrm{CBC}$ indices and $\mathrm{MCV}$ were normal $[\mathrm{Hb} / \mathrm{Hct}=14 \mathrm{mg} / \mathrm{dl}$ and $44 \%$ and $\mathrm{MCV}=92 \mathrm{fl}]$. A peripheral smear showed no hypersegmented neutrophils or macroovalocytes.
The patient's vitamin B12 level was $202 \mathrm{pg} / \mathrm{ml}$ (normal 180-900). Because the level was in the low normal range, we decided to send out blood for methymalonyl CoA and homocysteine levels which are known to be elevated even when the B12 level registers in the low normal range. Methylmalonyl CoA was $1078 \mathrm{nmol} / \mathrm{L}$ (normal 90-279), and homocysteine was $48.4 \mathrm{mc}-$ $\mathrm{mol} / \mathrm{L}$ (normal 5-15). We decided to treat him intramuscularly with 1000 micrograms of Vitamin B12 every day for one week and with low dose Quetiapine fumarate (Seroquel $25 \mathrm{mg}$ PO every day). He was discharged with instructions to take $1000 \mathrm{mi}-$ crograms, intramuscularly, of vitamin B12 every week for one month and advised to follow-up in the psychiatry out-patient clinic. At the time of discharge, 2 weeks after his presentation to our hospital, his fixed delusions started resolving and he felt better. He was lost to follow-up thereafter.

\section{DISCUSSION}

Nitrous oxide is a commonly abused inhalant drug due to its easy availability. It causes inactivation of vitamin B12 by the oxidation of Cob (I) alamin to Cob (III) alamin and can cause functional vitamin B12 deficiency, especially in people who have marginal B12 stores [2]. Vitamin B12 refers to any of the biologically active forms related to cyanocobalamin [1]. It is a complex molecule composed of a cobalt atom contained in a corrin ring, a tetrapyrrole structure similar to heme but with a cobalt atom rather than iron at its center [Figure 1].

Humans are completely dependent on dietary sources for cobalamin. Animal proteins such as red meat, poultry, eggs and

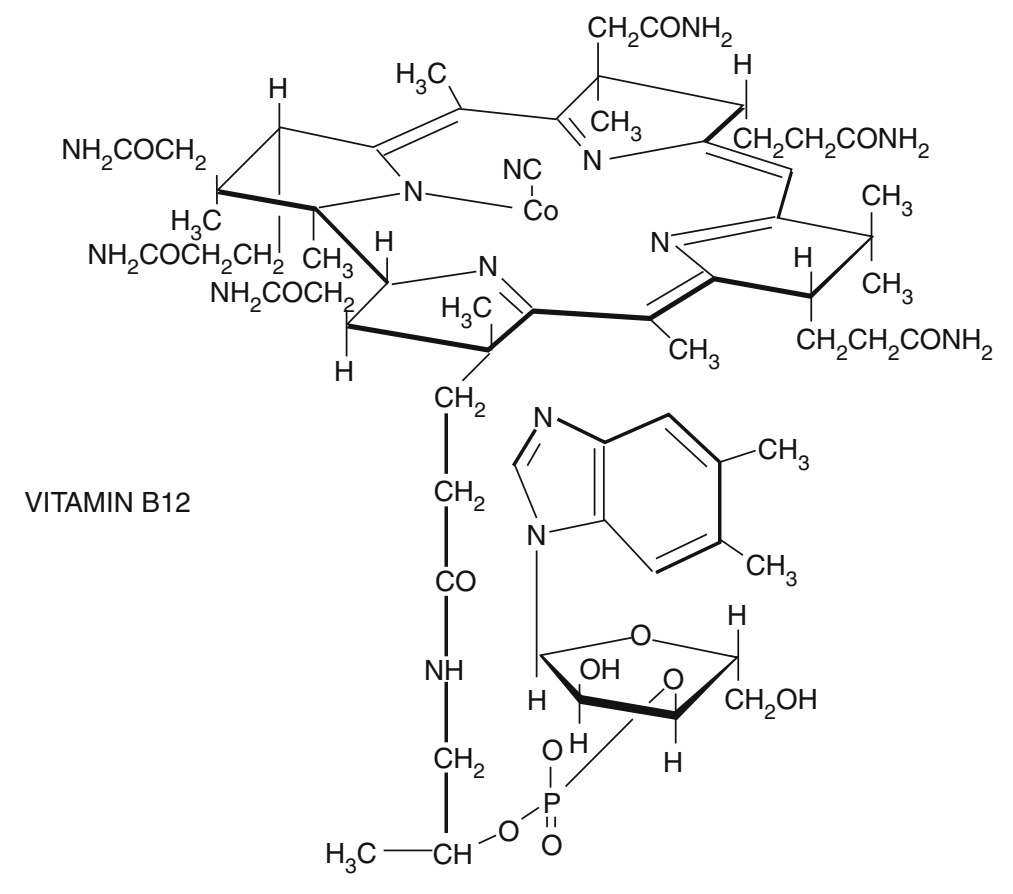

Figure 1. Structure of cobalamin. 

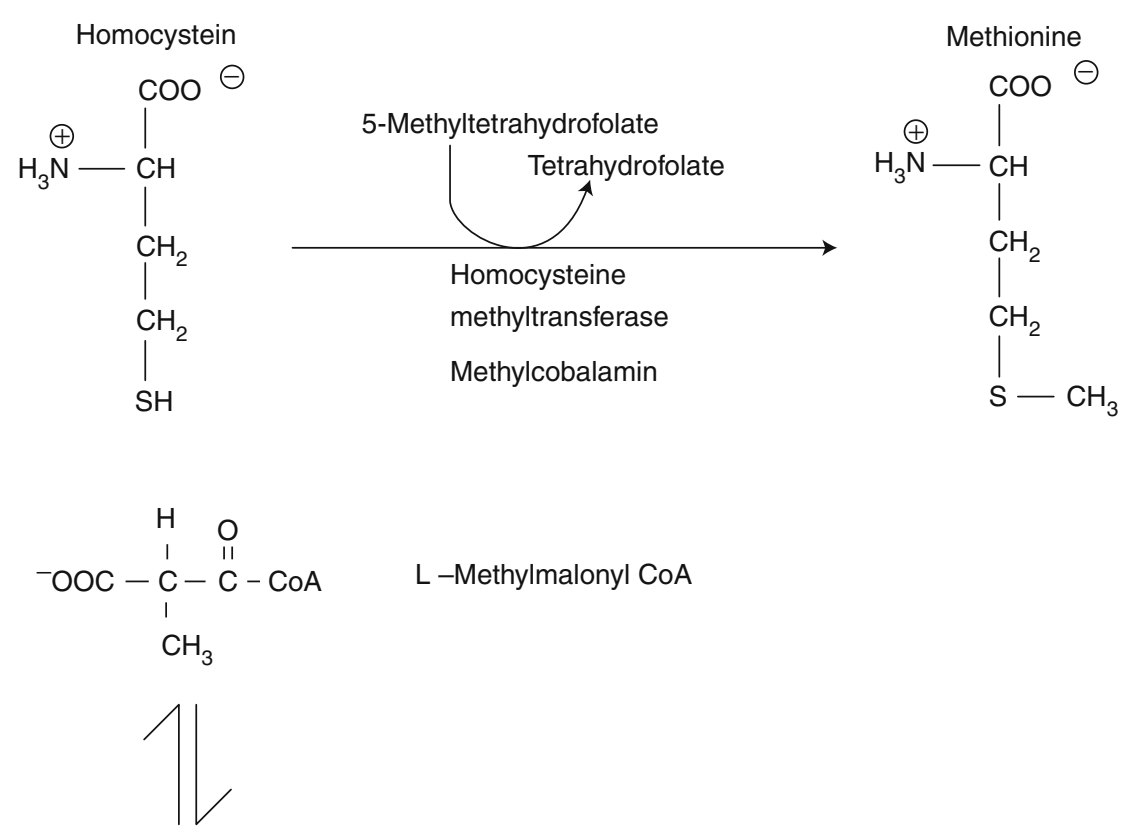

$\mathrm{L}$-Methylmalonyl CoA

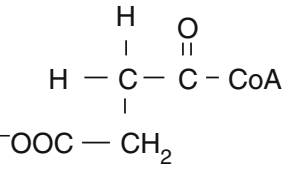

Succinyl CoA

Figure 2. Enzymatic reactions mediated by cobalamin.

dairy products provide rich sources of Vitamin B12; so deficiency is more frequent in strict vegetarians (vegans) than ovolactovegetarians [3]. The liver stores cyanocobalamin, and total body stores range from 2 to $10 \mathrm{mg}$. The recommended daily allowance (RDA) is $2.4 \mathrm{micrograms} /$ day in adults and $2.6 \mathrm{micrograms} /$ day in pregnant and lactating women. Cyanocobalamin mediates two important enzymatic reactions in humans [1]. The first reaction is the conversion of methylmalonyl-coenzyme A to succinylcoenzyme A. The second is the conversion of homocysteine to methionine [Figure 2].

A deficiency of cyanocobalamin leads to accumulation of methylymalonyl-CoA and homocysteine in the serum and these can be used as surrogate markers of vitamin B12 deficiency, the very chain of events that happened with the patient in our case. Apart from nitrous oxide protean, other conditions can cause B12 deficiency, including: pernicious anemia (absence or deficiency of intrinsic factor) various malabsorption syndromes (such as bacterial overgrowth, blind loop, post ileal resection, Crohn's disease, fish tapeworm, chronic infections like HIV, and drugs causing mucosal defects) and rarer congenital conditions such as transcobalamin deficiency [3].

Patients with Vitamin B12 deficiency may be entirely asymptomatic or present with protean hematological and neuropsychiatric manifestations. The various neurological manifestations vary from CNS manifestations-such as dementia, depression and cerebrovascular disease (homocysteine is an independent risk factor for strokes), spinal cord manifestations (sub acute combined degeneration of spinal cord) - to PNS manifestations (motor-sensory polyneuropathy) [1,4]. Neuropsychiatric manifestations (such as memory loss, depression, hypomania, paranoid psychosis with auditory and visual hallucinations which together comprise the so called "megaloblastic madness") have been described with vitamin B12 deficiency [5]. Patients may present with violent behavior or more subtle personality changes [6]. They may also present with vague complaints typical of agingsuch as fatigue, generalized weakness, and loss of memory. Cognitive testing may reveal frank dementia. These complaints may be attributed to aging unless a high index of suspicion is entertained and evaluated. Miller et al. reported a case of vitamin B12 deficiency due to nitrous oxide abuse presenting with ataxia and made a case for emergency physicians to recognize this not so commonly remembered "drug of abuse" [2].

Severe neurological impairment due to nitrous oxide inhalation has been reported in an infant during anesthesia [7]. "Whippits" are small cylindrical metal bulbs with a pierceable end that contain compressed nitrous oxide. These canisters are intended for use in whipped cream makers as propellants [2]. However, the cartridge may instead be attached via a "cracker" that is used to puncture the end, with the escaping gas captured in an inflatable object, such as a balloon, and ready for inhalation. Our patient 
had devised a unique contraption; he discharged the "whippits" into a container and then directly inhaled via a facemask.

We believe that his frequent use of nitrous oxide led to depletion of his marginal B12 stores and the development of neuropsychiatric manifestations of vitamin B12 deficiency. These may occur in the absence of the more typical neurological and hematological abnormalities. Treatment with parenteral vitamin B12 usually leads to complete recovery, as occurred with the patient in our case.

This case report highlights the need to consider vitamin B12 deficiency in patients who arrive at a hospital with psychiatric manifestations who report having history of nitrous oxide exposure or abuse in the recent or remote past.

The authors have no potential financial conflicts of interest to report.

\section{REFERENCES}

1. Herbert V. Vitamin B12-an overview. In Herbert V, ed Vitamin $\mathrm{B}_{12}$ deficiency. London: Royal Society of Medicine Press, 1999; $1-81$.
2. Miller MA, Martinez V, McCarthy R, et al. Nitrous oxide "whippet" abuse presenting as clinical B12 deficiency and ataxia. Am J Em Med. Mar 2004; 22(2)124.

3. Swain R. An update of vitamin $\mathrm{B}_{12}$ metabolism and deficiency states. J Fam Pract 1995; 41(6):595-600.

4. Chiew IS, Goh KL, Loh TG. Vitamin B-12 neuropathy in the absence of anemia-case report. Singapore Med J.Apr 1989; 30(2):221-2.

5. Lindenbaum J, Healton EB, Savage DG, et al. Neuropsychiatric disorders caused by cobalamin deficiency in the absence of anemia or macrocytosis. N Engl J Med 1988; 318(26):1720-8.

6. Masalha R, Chudakov B, Muhamad M, et al. Cobalamine responsive psychosis as the sole manifestation of vitamin B12 deficiency. IMAJ 2001; 3:701-703.

7. McNeely JK, Buczulinski B, Rosner DR. Severe neurological impairment in an infant after nitrous oxide inhalation. Anesthesiology. Dec 2000; 93(6):1549-50. 